\title{
Energy-Momentum Tensor for the Electromagnetic Field in a Dielectric
}

\author{
Michael E. Crenshaw and Thomas B. Bahder \\ RDMR-WSS, Aviation and Missile RDEC, US Army RDECOM, Redstone Arsenal, AL 35898, USA
}

(Dated: October 22, 2018)

\begin{abstract}
The total momentum of a thermodynamically closed system is unique, as is the total energy. Nevertheless, there is continuing confusion concerning the correct form of the momentum and the energy-momentum tensor for an electromagnetic field interacting with a linear dielectric medium. Rather than construct a total momentum from the Abraham momentum or the Minkowski momentum, we define a thermodynamically closed system consisting of a propagating electromagnetic field and a negligibly reflecting dielectric and we identify the Gordon momentum as the conserved total momentum by the fact that it is invariant in time. In the formalism of classical continuum electrodynamics, the Gordon momentum is therefore the unique representation of the total momentum in terms of the macroscopic electromagnetic fields and the macroscopic refractive index that characterizes the material. We also construct continuity equations for the energy and the Gordon momentum, noting that a time variable transformation is necessary to write the continuity equations in terms of the densities of conserved quantities. Finally, we use the continuity equations and the time-coordinate transformation to construct an array that has the properties of a traceless, symmetric energy-momentum tensor.
\end{abstract}

\section{INTRODUCTION}

The energy-momentum tensor is a concise way to represent the conservation properties of an unimpeded flow field. For most types of simple flows, the energymomentum tensor is well-defined, with the notable exception of the electromagnetic field in a linear dielectric material. The Abraham-Minkoswski controversy [1 9 ] for the momentum of electromagnetic fields in a dielectric began with the derivation of the energy-momentum four-tensor by Minkowski [10]. Noting that the unsymmetrical Minkowski tensor does not support conservation of angular momentum, Abraham [11] proposed an energy-momentum tensor that was symmetric, but at the expense of a new phenomenological force. In order to address this constraint and additional issues, Einstein and Laub [7], Nelson [8], and others proposed variants of the energy-momentum tensor.

The crux of the Abraham-Minkowski controversy is whether the electromagnetic momentum density in a dielectric is of the Minkowski form

$$
\mathbf{g}_{M}=\frac{1}{c}(\mathbf{D} \times \mathbf{B})
$$

or the Abraham form

$$
\mathbf{g}_{A}=\frac{1}{c}(\mathbf{E} \times \mathbf{H}) .
$$

Experimental efforts to resolve the theoretical impasse have not been definitive. While some experiments favor the Abraham formula, other experiments support Minkowski's version. Brevick's [12] analysis of experiments performed by Jones and Richards 13], Ashkin and Dziedzic [14], and others showed that the allocation of momentum between the field and material was the determining factor in whether a particular experimental result was described by the Abraham or Minkowski form of electromagnetic momentum. Following Brevick [12], the formula for the field momentum has been shown repeatedly to be arbitrary such that any of the formulas for the field momentum can be combined with an appropriate momentum for the material to produce the same total momentum [15-17].

In 1973, Gordon [1] constructed the total momentum from a microscopic model in which the electromagnetic field component of the total momentum is said to be the Abraham momentum and the dielectric is treated as a dilute collection of electric dipoles with center-of-mass motion in the direction of propagation of the field. Gordon [1] discusses the empirical and experimental validation of the total momentum density

$$
\mathbf{g}_{G}=\frac{n}{c}(\mathbf{E} \times \mathbf{B})
$$

and shows that the density $\mathbf{g}_{G}$, integrated over a volume containing the entire field, is invariant in time. Gordon concludes that the Abraham momentum density represents the true momentum density of the electromagnetic field and the Minkowski momentum density includes a pseudomomentum.

In this article, we investigate conservation of energy and momentum in a thermodynamically closed system consisting of the macroscopic electromagnetic field and a negligibly reflecting linear dielectric (such as a dielectric with an anti-reflection coating). Here, we identify the Gordon momentum 1] as the total momentum by the fact that it is invariant in time and therefore a conserved quantity in an isolated system. We find that it is not necessary to decompose the Gordon momentum into the sum of a field momentum, such as the Abraham or Minkowski momentum, and a material momentum, such as the canonical or kinetic momentum 5, 17]. Instead, we work with the total energy and total momentum and derive continuity equations in terms of the densities of these conserved quantities. The continuity equations are then used to construct an array that has the properties 
of a traceless, symmetric energy-momentum tensor, but in a coordinate system with time-like coordinate $c t / n$.

\section{ENERGY-MOMENTUM TENSOR OF NONINTERACTING PARTICLES}

In the continuum limit, the density of any property of identical noninteracting particles can be treated as the number density multiplied by the amount of the property that can be attributed to each particle. The continuity equation corresponding to a specific property, such as mass, charge, or energy, is then obtained by substitution of the specific property density for a placeholder number density. For an infinitesimal element of volume in an inviscid sourceless flow, the continuity equation

$$
\frac{\partial s}{\partial t}+\nabla \cdot s \mathbf{u}=0
$$

is derived by applying the divergence theorem to a Taylor series expansion of the property density field $s$ and the vector velocity field $\mathbf{u}=\left(u^{x}, u^{y}, u^{z}\right)$ of the flow [18]. The continuity equation reflects the conservation of a continuous scalar property in a flow in terms of the equality of the net rate of flux out of the volume and the time rate of change of the property density $s$ inside the volume. Depending on the context, the three-vector

$$
\mathbf{g}=s \mathbf{u}
$$

is known as the momentum density, the flux, or the current density of the property. Specifically, $\mathrm{g}$ corresponds to the linear momentum density if $s$ is a mass density and corresponds to the charge current density if $s$ is the electric charge density.

Some conserved properties, such as momentum, are vectors. For a flow, the density of a conserved vector property can be represented as $\mathbf{s}=\left(s^{x}, s^{y}, s^{z}\right)$. Applying the scalar formalism to the three orthogonal components of the property density vector yields scalar continuity equations

$$
\begin{aligned}
& \frac{\partial s^{x}}{\partial t}+\nabla \cdot s^{x} \mathbf{u}=0 \\
& \frac{\partial s^{y}}{\partial t}+\nabla \cdot s^{y} \mathbf{u}=0 \\
& \frac{\partial s^{z}}{\partial t}+\nabla \cdot s^{z} \mathbf{u}=0 .
\end{aligned}
$$

At this point, we adopt a four-dimensional notation where repeated indices are summed. We take Roman indices to run from 1 to 3 and we identify the coordinates $x^{i}$ with the Cartesian coordinates, such that $x^{1}=x$, $x^{2}=y$, and $x^{3}=z$. Greek indices run from 0 to 3 and $x^{0}$ is identified with the time-like coordinate $c t$. The Minkowski space-time metric is $\operatorname{diag}(-1,1,1,1)$. Finally, partial differentiation with respect to the indexed coordinates is represented by $\partial_{\alpha}=\partial /\left(\partial x^{\alpha}\right)$.

The four continuity equations (4) and (6) can be concisely represented by

$$
\partial_{\beta} G^{\alpha \beta}=0
$$

where

$$
G^{\alpha \beta}=\left[\begin{array}{cccc}
c^{2} s & c u^{x} s & c u^{y} s & c u^{z} s \\
c s^{x} & u^{x} s^{x} & u^{y} s^{x} & u^{z} s^{x} \\
c s^{y} & u^{x} s^{y} & u^{y} s^{y} & u^{z} s^{y} \\
c s^{z} & u^{x} s^{z} & u^{y} s^{z} & u^{z} s^{z}
\end{array}\right]
$$

Consider a transformation to a new set of coordinates $x^{i^{\prime}}=f_{i}\left(x^{0}, x^{1}, x^{2}, x^{3}\right)$. In order for this matrix to transform as a tensor, $s^{x}, s^{y}$, and $s^{z}$ must be expressible in terms of $s$ and components of $\mathbf{u}$. For a closed system, conservation of angular momentum requires $G^{\alpha \beta}$ to be symmetric [19]

$$
G^{\alpha \beta}=G^{\beta \alpha}
$$

Therefore, the vector property s must represent the flux of the conserved scalar quantity $s$ such that $\mathbf{s}=s \mathbf{u}$. Conversely, the flux $s \mathbf{u}$ of a scalar property of the particles is a conserved vector property of the flow. The covariant form of the continuity equation is the four-divergence, Eq. (7), of the energy-momentum four-tensor

$$
G^{\alpha \beta}=\left[\begin{array}{cccc}
c^{2} s & c s u^{x} & c s u^{y} & c s u^{z} \\
c s u^{x} & s u^{x} u^{x} & s u^{x} u^{y} & s u^{x} u^{z} \\
c s u^{y} & s u^{y} u^{x} & s u^{y} u^{y} & s u^{y} u^{z} \\
c s u^{z} & s u^{z} u^{x} & s u^{z} u^{y} & s u^{z} u^{z}
\end{array}\right]
$$

The continuity equation (7) with the four-tensor (10) is valid for any conserved extensive quantity in a simple flow, not just for the mass of a fluid.

The energy-momentum tensor given in Eq. (10) has some essential properties. First, the four-divergence of each row vector, Eq. (7), is a continuity law corresponding to the conservation of the property represented by the property density in the first element of that row. Second, the tensor is symmetric, corresponding to the absence of unbalanced shear forces and conservation of angular momentum in a closed system [19]. Third, as a consequence of diagonal symmetry,

$$
\partial_{\alpha} G^{\alpha \beta}=0
$$

the four-divergence of each column vector is a continuity law corresponding to the conservation of the property represented by the property density in the first element of that column. It should also be noted that this simple energy-momentum tensor is based on the properties of an unimpeded flow. If the flow is redirected by impact with a macroscopic object then one is obligated to include the equations of motion of the object or the forces of restraint. 


\section{THE ABRAHAM AND MINKOWSKI ENERGY-MOMENTUM TENSORS}

The Abraham and Minkowski energy-momentum tensors are examples of a number of different tensors that have been proposed for the electromagnetic field in a dielectric [15]. The Minkowski tensor can be constructed from the continuity equations for energy flux and momentum flux by the same procedure that was used to construct the array (8). The Minkowski tensor is not symmetric and therefore violates angular momentum conservation if it is the total energy-momentum tensor of a closed system. Consequently, the Minkowski tensor is considered to be a representation of the energy and momentum of a component of the system [15]. In this section, we outline the construction of the Minkowski tensor and describe how the procedure is modified to obtain the Abraham tensor. Neither the Minkowski tensor, nor the Abraham tensor, satisfy the requirements of a total energy-momentum tensor. The Minkowski tensor is not symmetric while the Abraham tensor contains a phenomenological volume force.

The macroscopic Maxwell equations of continuum electrodynamics are the basis for deriving continuity equations for electric and magnetic fields in a dielectric. For a dielectric with no free charges in a regime of negligible absorption and dispersion, the Maxwell equations may be written as

$$
\begin{gathered}
\nabla \times \mathbf{E}=-\frac{\partial \mathbf{B}}{\partial(c t)} \\
\nabla \times \mathbf{B}=\frac{\partial n^{2} \mathbf{E}}{\partial(c t)} \\
\nabla \cdot \mathbf{B}=0 \\
\nabla \cdot \frac{n^{2}}{c^{2}} \mathbf{E}=0
\end{gathered}
$$

in Heaviside-Lorentz units. The electric and magnetic fields can be defined in terms of the vector potential $\mathbf{A}$ as

$$
\begin{aligned}
& \mathbf{E}=-\frac{\partial \mathbf{A}}{\partial(c t)} \\
& \mathbf{B}=\nabla \times \mathbf{A}
\end{aligned}
$$

for transverse fields in the Coulomb gauge.

The macroscopic Maxwell equations are the axioms of classical continuum electrodynamics. Poynting's theorem,

$$
\frac{\partial}{\partial(c t)}\left[\frac{1}{2}\left(n^{2} \mathbf{E}^{2}+\mathbf{B}^{2}\right)\right]+\nabla \cdot(\mathbf{E} \times \mathbf{B})=0,
$$

can be derived by multiplying the Faraday law (12a) by $\mathbf{B}$ and adding it to the Maxwell-Ampère law (12b) multiplied by E. Poynting's theorem can also be derived by substituting the Maxwell equations into the temporal derivative of the energy density

$$
\rho_{e}=(1 / 2)\left(n^{2} \mathbf{E}^{2}+\mathbf{B}^{2}\right)
$$

using a vector triple-product identity. Poynting's theorem is a continuity equation for the Poynting energy-flux vector

$$
\mathbf{S}_{P}=c(\mathbf{E} \times \mathbf{B})=\left(s_{P}^{1}, s_{P}^{2}, s_{P}^{3}\right)
$$

The theorem

$$
\begin{gathered}
\frac{\partial}{\partial(c t)}\left(n^{2} \mathbf{E} \times \mathbf{B}\right)=-\mathbf{B} \times(\nabla \times \mathbf{B})+\mathbf{B}(\nabla \cdot \mathbf{B}) \\
-n^{2} \mathbf{E} \times(\nabla \times \mathbf{E})+\mathbf{E}\left(\nabla \cdot n^{2} \mathbf{E}\right)
\end{gathered}
$$

is derived, in a manner similar to Poynting's theorem, by substituting Maxwell's equations into the temporal derivative of the Minkowski momentum density, $\mathbf{g}_{M}=$ $\left(n^{2} / c\right)(\mathbf{E} \times \mathbf{B})$. The right-hand side of Eq. (17) can be recast, approximately, as the negative of the divergence of the Maxwell stress tensor [20] with components

$$
W^{i j}=\left[-n^{2} E_{i} E_{j}-B_{i} B_{j}+\frac{1}{2}\left(n^{2} \mathbf{E} \cdot \mathbf{E}+\mathbf{B} \cdot \mathbf{B}\right) \delta_{i j}\right],
$$

where terms involving the gradient of $n^{2}$ have been neglected. Then the temporal derivative of the Minkowski momentum density, Eq. (17), can be expressed using the vector divergence operator, $\boldsymbol{\nabla} \cdot$, as

$$
\frac{\partial}{\partial(c t)}\left(n^{2} \mathbf{E} \times \mathbf{B}\right)+\boldsymbol{\nabla} \cdot \mathbf{W}=0 .
$$

The array

$$
T_{M}^{\alpha \beta}=\left[\begin{array}{cccc}
\rho_{e} & s_{P}^{1} / c & s_{P}^{2} / c & s_{P}^{3} / c \\
c g_{M}^{1} & W^{11} & W^{12} & W^{13} \\
c g_{M}^{2} & W^{21} & W^{22} & W^{23} \\
c g_{M}^{3} & W^{31} & W^{32} & W^{33}
\end{array}\right]
$$

known as the Minkowski energy-momentum tensor, is constructed from continuity equations (14) and (19). Using the summation convention, we can write Poynting's theorem in Eq. (14) as

$$
\partial_{0} T_{M}^{00}+\partial_{j} T_{M}^{0 j}=0
$$

for continuity of the energy flux and Eq. (19) as

$$
\partial_{0} T_{M}^{i 0}+\partial_{j} T_{M}^{i j}=0
$$

for continuity of the momentum flux. Then, each row of $T_{M}$ corresponds to a four-divergence

$$
\partial_{\beta} T_{M}^{\alpha \beta}=0 .
$$


Because the array $T_{M}^{\alpha \beta}$ in Eq. (20) is not symmetric, Abraham proposed the energy-momentum tensor

$$
T_{A}^{\alpha \beta}=\left[\begin{array}{cccc}
\rho_{e} & s_{P}^{1} / c & s_{P}^{2} / c & s_{P}^{3} / c \\
c g_{A}^{1} & W^{11} & W^{12} & W^{13} \\
c g_{A}^{2} & W^{21} & W^{22} & W^{23} \\
c g_{A}^{3} & W^{31} & W^{32} & W^{33}
\end{array}\right],
$$

where $\mathbf{g}_{A}$ is given by Eq. (2). The four divergence of this tensor is

$$
\partial_{\beta} T_{A}^{\alpha \beta}=-f^{\beta},
$$

where $f^{\beta}$ is the Abraham force. The Cartesian components of the Abraham force

$$
\mathbf{f}=\frac{\partial}{\partial(c t)}\left(\left(n^{2}-1\right) \mathbf{E} \times \mathbf{B}\right)
$$

are obtained by substituting the Abraham momentum density into the continuity equation (19) and the timelike coordinate component is $f^{0}=0$.

It has been widely reported in the literature that neither $T_{M}$ nor $T_{A}$ is the total energy-momentum tensor [15]. Instead, they are to be considered two of many arbitrary forms of the electromagnetic part, $T_{\mathrm{fld}}$, of a total energy momentum tensor

$$
T=T_{\text {fld }}+T_{\text {matl }}
$$

composed of energy-momentum tensors for the field and material subspaces, however those subspaces are defined.

\section{TOTAL MOMENTUM}

A well-defined quantity for the total momentum is only derivable from the macroscopic Maxwell equations by imposing an additional condition: The total momentum of an isolated system must be constant in time. In this section, we identify the unique total momentum using this constraint. We consider the case of a quasimonochromatic electromagnetic field, in the plane-wave limit, entering a linear medium from vacuum at normal incidence. The medium is taken to be a simple linear dielectric in the regime of negligible dispersion and negligible absorption. In the absence of reflection, there is no momentum given to the dielectric slab and it remains stationary. We adopt this case of a stationary dielectric in which reflections can be neglected by assuming that an antireflection coating has been applied to the dielectric or that the refractive index of the dielectric is only slightly greater than unity.

Gordon [1] used a microscopic model of the dielectric as a vapor of weakly polarizable atoms and derived the material momentum as the continuum average of the mechanical momentum of the atoms. Assuming a rarefied vapor of atoms in order for reflections to be negligible, Gordon obtained the total momentum

$$
\mathbf{G}_{\mathrm{G}}=\int_{V} d v \mathbf{g}_{G}=\int_{V} d v \frac{n}{c}(\mathbf{E} \times \mathbf{B})
$$

by adding the material momentum to the Abraham momentum. Here the integration is over all threedimensional space. In continuum electrodynamics, the electrodynamic properties of a material are characterized only by a macroscopic refractive index. Therefore, the microscopic origin of the Gordon momentum (28) is of no consequence in the formalism of continuum electrodynamics. Following Gordon, we will demonstrate that the momentum (28) is invariant in time and, because it is conserved, can be identified as the total momentum. Because the Gordon momentum depends on the material only through the refractive index, it is the unique total momentum for all cases in which the medium behaves, to a good approximation, as a negligibly reflecting linear dielectric with refractive index $n$.

Propagation of a field in a linear medium is governed by the wave equation,

$$
\nabla^{2} \mathbf{A}-\frac{n^{2}}{c^{2}} \frac{\partial^{2} \mathbf{A}}{\partial t^{2}}=0
$$

written in terms of the vector potential $\mathbf{A}$, where $\mathbf{B}=$ $\nabla \times \mathbf{A}$. For quasi-monochromatic plane waves, it is convenient to write the vector potential in terms of a slowly varying envelope function, $A(z, t)$, a rapidly varying carrier, and a unit vector, $\mathbf{e}_{\mathbf{k}}$, in the direction of propagation as $\mathbf{A}=A(z, t) e^{-i\left(\omega_{0} t-k z\right)} \mathbf{e}_{\mathbf{k}}$. For a plane-wave entering a dielectric at normal incidence, reflections are negligible if $\delta n=n-1$ is small. In this limit, there is no momentum given to the bulk material, which remains stationary, and we can apply the Fresnel relation

$$
\frac{A_{t}}{A_{i}}=\frac{2}{n+1}=\left(1+\frac{\delta n}{2}\right)^{-1}
$$

where $A_{i}$ is the incident amplitude and $A_{t}$ is the transmitted amplitude. Comparing Eq. (30) with a series expansion of $1 / \sqrt{n}$ in the limit of small $\delta n$, we find that the vector potential amplitude inside the dielectric, $A_{t}$, is reduced by a factor of $\sqrt{n}$ from the incident amplitude $A_{i}$.

For continuous plane waves, and approximately for slowly varying waves, the relation between the amplitudes of the fields simplifies to

$$
|\mathbf{B}|=n|\mathbf{E}|=\frac{n \omega}{c}|\mathbf{A}| .
$$

Then, the electromagnetic energy density, $\rho_{e}=$ $(1 / 2)\left(n^{2} \mathbf{E}^{2}+\mathbf{B}^{2}\right)$, can be written as

$$
\rho_{e}=\frac{n^{2} \omega^{2}}{c^{2}}|\mathbf{A}|^{2} .
$$

Applying the Fresnel amplitudes to relate the fields inside and outside the medium, $A_{t}=A_{i} / \sqrt{n}$, we find that the energy density inside the material is a factor of $n$ larger than the energy density of the same field in the vacuum. The region occupied by the field in the material is compressed spatially by a factor of $n$ due to the 
reduced speed of light within the medium, such that the total energy

$$
U=\int_{V} d v \rho_{e}=\int_{V} d v \frac{n^{2} \omega^{2}}{c^{2}}|\mathbf{A}|^{2}
$$

is conserved. Numerical solutions of the wave equation for a field entering a linear material through a gradientindex anti-reflection coating indicate that the field in the material is a factor of $\sqrt{n}$ smaller and a factor of $n$ narrower than the field in the vacuum, independent of the magnitude of $n$, as long as reflections are suppressed [21].

Having demonstrated the conservation properties of the electromagnetic energy, we demonstrate the conservation properties of the Gordon electromagnetic momentum by a similar procedure. The Gordon momentum [1] is obtained by integrating the momentum density (3) over all three-dimensional space. Comparing the Gordon momentum, expressed in terms of the envelope functions

$$
\mathbf{G}_{\mathrm{G}}=\int_{V} d v \frac{n}{c}(\mathbf{E} \times \mathbf{B})=\int_{V} d v \frac{n^{2} \omega^{2}}{c^{3}}|\mathbf{A}|^{2} \mathbf{e}_{k},
$$

with the total energy in Eq. (33), we see that conservation of total energy implies conservation of the Gordon momentum. The total momentum of a closed system is unique and the Gordon form of total momentum is conserved. Therefore, the Gordon momentum can be identified as the total momentum of the thermodynamically closed system. Because neither the Minkowski momentum nor the Abraham momentum is the total momentum, neither is conserved in a thermodynamically closed system.

We point out that, in the macroscopic limit in which the dielectric is described by a refractive index $n$, the question of what portion of the energy given by Eq. (33) resides in the field or dielectric is improperly posed. Comparing Eqs. (33) and (34), we see that the same holds true for the apportionment of the momentum into field and dielectric components.

\section{TOTAL ENERGY-MOMENTUM TENSOR}

In the previous section, we identified the unique total momentum for the system of an electromagnetic field in a dielectric for the case of negligible reflections. In this section, we construct the corresponding total energymomentum tensor from continuity equations for the energy and momentum.

A continuity equation is a differential form of a conservation law applied to an element of volume in a continuous flow. In Sec. II we showed that the energymomentum tensor for dust is constructed from continuity equations in which the differential operators act on the densities of conserved quantities. The operand of the time derivative in the continuity Eq. (19) is the Minkowski momentum density. Because the volume integral of the Minkowski momentum density is not a conserved vector quantity, we do not consider Eq. (19) to be a suitable continuity equation with which to construct an energy-momentum tensor. Instead, we write Eq. (19) as

$$
\frac{n}{c} \frac{\partial}{\partial t} c \mathbf{g}_{\mathrm{G}}+\mathbf{\nabla} \cdot \mathbf{W}=0
$$

in terms of the Gordon momentum density in Eq. (3) whose volume integral is a conserved vector quantity. Equation (35) provides three continuity equations for our energy-momentum tensor. The additional continuity equation is obtained by writing Poynting's theorem in (14) as

$$
\frac{n}{c} \frac{\partial \rho_{e}}{\partial t}+\nabla \cdot[n(\mathbf{E} \times \mathbf{B})]=\frac{\nabla n}{n} \cdot n(\mathbf{E} \times \mathbf{B})
$$

using the densities of conserved quantities. Again, we are considering the case of a closed system in which there are no reflections. As this is assumed to be accomplished by a gradient-index antireflection coating, we can drop the term containing $\nabla n$ and write Poynting's theorem in Eq. (36) as

$$
\frac{n}{c} \frac{\partial \rho_{e}}{\partial t}+\nabla \cdot[n(\mathbf{E} \times \mathbf{B})]=0 .
$$

In Maxwell's equations, expressed in terms of 3vectors, time is not a coordinate. We make a simple change of time variable to $\tau=t / n$ and write the continuity theorems (35) and (37) as

$$
\begin{gathered}
\frac{1}{c} \frac{\partial}{\partial \tau} c \mathbf{g}_{\mathrm{G}}+\boldsymbol{\nabla} \cdot \mathbf{W}=0 \\
\frac{1}{c} \frac{\partial \rho_{e}}{\partial \tau}+\nabla \cdot c \mathbf{g}_{G}=0,
\end{gathered}
$$

where the Gordon momentum density $\mathbf{g}_{G}$ is given in Eq. (3). However when writing Maxwell's equations as tensor equations, time is one of the four space-time coordinates and we define the time-like coordinate

$$
\bar{x}^{0}=c \tau=\frac{c t}{n} .
$$

Then the four scalar continuity equations, Eqs. (38a) and (38b), can be written concisely as a single equation, as in Section 2, defining the operator

$$
\bar{\partial}_{\alpha}=\left(\frac{\partial}{\partial \bar{x}^{0}}, \partial_{x}, \partial_{y}, \partial_{z}\right)
$$

and an array

$$
T^{\alpha \beta}=\left[\begin{array}{cccc}
\rho_{e} & c g_{\mathrm{G}}^{1} & c g_{\mathrm{G}}^{2} & c g_{\mathrm{G}}^{3} \\
c g_{\mathrm{G}}^{1} & W^{11} & W^{12} & W^{13} \\
c g_{\mathrm{G}}^{2} & W^{21} & W^{22} & W^{23} \\
c g_{\mathrm{G}}^{3} & W^{31} & W^{32} & W^{33}
\end{array}\right],
$$

such that

$$
\bar{\partial}_{\beta} T^{\alpha \beta}=0
$$


The array that appears in Eq. (41) has a number of notable properties. The array is symmetric

$$
T^{\alpha \beta}=T^{\beta \alpha}
$$

and has a vanishing trace

$$
T_{\alpha}^{\alpha}=0 .
$$

The operator defined in Eq. (40) applied to the rows of the array in Eq. (41) generates continuity laws for demonstrably conserved electromagnetic energy and momentum properties. Similarly, the operator in Eq. (40) applied to the columns,

$$
\bar{\partial}_{\alpha} T^{\alpha \beta}=0,
$$

generates the same continuity equations as a consequence of the symmetry of the array. These are the properties that we associate with an energy-momentum tensor. Ravndal [22] arrives at the same energy-momentum tensor as in Eq. (41) using symmetry arguments, but interprets the continuity equations, Eqs. (42), in the context of the Minkowski momentum, which we have shown is not conserved.

\section{CONCLUDING REMARKS}

For many years, the Abraham-Minkowski controversy has been resolved by postulating a total energy- momentum tensor that is comprised of separate field and matter tensors - an approach that involves assumptions about the behavior of matter in the presence of an electromagnetic field. In continuum electrodynamics the interaction of the field and matter is described in terms of a single macroscopic parameter, the refractive index $n$. We showed that the Gordon momentum is the total momentum of a thermodynamically closed system consisting of a quasimonochromatic field and negligibly reflecting linear dielectric in the continuum. We derived continuity equations from the Maxwell equations and used a time variable transformation to write the continuity equations in terms of densities of conserved energy and Gordon momentum quantities. When written in four-dimensional tensor form with time-like coordinate $c t / n$, the continuity equations are obtained from the four-divergence of a traceless, diagonally symmetric energy-momentum tensor.

In summary, for the case of an electromagnetic field and negligibly reflecting dielectric, we constructed the total energy-momentum tensor in Eq. (41) from continuity equations that were derived from the macroscopic Maxwell's equations. It is interesting to note that a time coordinate transformation was required in order to write the continuity equations as the four-divergence of the symmetric energy-momentum tensor.
[1] J. P. Gordon, "Radiation Forces and Momenta in Dielectric Media," Phys. Rev. A 8, 14-21 (1973).

[2] R. Peiels, "The momentum of light in a refracting medium," Proc. R. Soc. Lond. A 347, 475-491 (1976).

[3] M. Kranys, "The Minkowski and Abraham tensors, and the non-uniqueness of non-closed systems: resolution of the controversy," Int. J. Engng. Sci. 20, 1193-1213 (1982).

[4] Y. N. Obukhov and F. W. Hehl, "Electromagnetic energy-momentum and forces in matter," Phys. Lett. A 311, 277-284 (2003).

[5] J. C. Garrison and R. Y. Chiao, "Canonical and kinetic forms of the electromagnetic momentum in an ad hoc quantization scheme for a dispersive dielectric," Phys. Rev. A 70, 053826-1-053826-8 (2004).

[6] S. Antoci and L. Mihich, "A forgotten argument by Gordon uniquely selects Abraham's tensor as the energymomentum tensor for the electromagnetic field in homogeneous, isotropic matter," Nuovo Cim. B112, 991-1001 (1997).

[7] A. Einstein and J. Laub, "Über die im elektromagnetischen Felde auf ruhende Körper ausge" ubten ponderomotorischen Kräfte," Ann. Phys. (Leipzig) 26, 541-550 (1908).

[8] D. F. Nelson, Momentum, pseudomomentum, and wave momentum: Toward resolving the Minkowski-Abraham controversy," Phys. Rev. A 44, 3985-3996 (1991).

[9] P. Penfield, Jr. and H. A. Haus, Electrodynamics of Moving Media (MIT Press, 1967).

[10] H. Minkowski, Natches. Ges. Wiss. Göttingen 53 (1908).

[11] M. Abraham, Rend. Circ. Mat. Palermo 28, 1 (1909); 30, 33 (1910).

[12] I. Brevik, "Experiments in phenomenological electrodynamics and the electromagnetic energy-momentum tensor," Phys. Rep. 52, 133-201 (1979).

[13] R. V. Jones and J. C. S. Richards, "The pressure of radiation in a refracting medium," Proc. R. Soc. London A 221, 480-498 (1954).

[14] A. Ashkin and J. M. Dziedzic, "Radiation Pressure on a Free Liquid Surface," Phys. Rev. Lett. 30, 139-142 (1973).

[15] R. N. C. Pfeifer, T. A. Nieminen, N. R. Heckenberg, and H. Rubinsztein-Dunlop, "Colloquium: Momentum of an electromagnetic wave in dielectric media," Rev. Mod. Phys. 79, 1197-1216 (2007).

[16] Z. Mikura, "Variational formulation of the electrodynamics of fluids and its application to the radiation pressure problem," Phys. Rev. A 13, 2265-2275 (1976).

[17] S. M. Barnett, "Resolution of the Abraham-Minkowski Dilemma," Phys. Rev. Lett. 104, 070401-1-070401-4

[18] See for example: R. W. Fox and A. T. McDonald, Introduction to Fluid Dynamics, 2nd. ed. (Wiley, 1978). 
[19] L. D. Landau and E. M. Lifshitz, The Classical Theory of Fields, 4th. ed., (Elsevier, 2006).

[20] J. D. Jackson, Classical Electrodynamics, 2nd ed., (Wiley, 1975).

[21] M. E. Crenshaw, "Electromagnetic momentum and radi- ation pressure derived from the Fresnel relations," Opt. Exp. 15, 714 (2007).

[22] F. Ravndal, "Electromagnetic energy-momentum tensors in media," arXiv:0805.2606 v2 (2008). 